\title{
Dante "Mistico"?*
}

Come si sa, la critica dantesca è profondamente divisa sulla relazione che intercorre tra teologia (intesa nel senso patristico, come esperienza religiosa) e poesia. Da una parte, il vecchio crocianesimo mal disposto a indagare motivi che non siano "squisitamente poetici," o comunque tendente a escludere il motivo religioso come in ogni caso indecifrabile (di qui la svalutazione del Paradiso rispetto alle altre cantiche). Dall'altra le letture che fanno del motivo religioso, dell'esperienza religiosa, l'ispirazione centrale della Commedia. Come è noto, per alcuni critici (Nardi, Sarolli), Dante parla con voce consapevolmente profetica; per altri (Singleton), si propone di imitare il modo di scrivere di Dio. Altri ancora, meno ambiziosamente, spiegano la particolarità della poesia di Dante richiamandosi a un'esperienza religiosa, intesa in senso psicologico, comune a tutti i credenti ma espressa nella Commedia con particolare efficacia "poetica." Così per esempio Giovanni Getto: "Questo sentimento della Grazia come gioia profonda che fa trasalire l'anima, come interiore pace che inonda il cuore, fatto ricco di un improvviso dono e di un'ignota ricchezza, è per l'appunto il sentimento teologico e nello stesso tempo umanissimo, su cui Dante imposta la sua massima espressione poetica" (199). Lo stesso contenutismo che affianca una teologia e una poesia quanto mai indefinibili si trova nel Fallani, di cui cito un passaggio tipico: "dopo la mistica folgorazione, si placa il mondo dottrinale, la cultura e la storia; ogni prospettiva dantesca ha ritrovato al di fuori di sè l'ordine dell'universo, dentro di sè la beatitudine di Dio" (244). Comune alle posizioni solo apparentemente opposte della critica, diciamo così, laica e di quella cattolica, è l'assegnare l'esperienza religiosa, o, in senso generico, mistica, e l'esperienza poetica, o, meglio, il fare poesia, ai due tradizionali e opposti versanti del contenuto e della forma. L'"interiore pace che inonda il cuore" è un sentimento, un contenuto su cui il poeta "imposta" la sua "espressione poetica," forma intoccabile che ha il solo difetto di adattarsi male, vestito troppo corto e troppo stretto, al "dono" e alla "ricchezza" soggiacenti. 
Prima di procedere oltre, conviene intendersi sul termine "mistica." Per il Dictionnaire de spiritualité ascétique et mystique, il fenomeno mistico è "in primo luogo un movimento per superare se stessi in direzione di un oggetto particolare, nè semplicemente profano, nè eterno, ma situato al di là dei limiti dell'esperienza normale, empirica"; "in secondo luogo, . . . la percezione intuitiva di questo oggetto o di questo essere, sia già caratterizzato da tratti personali, sia in condizione di entità profonda o cosmica" (voce "mystique"). Vorrei attenermi alla genericità di questa definizione che ci risparmia l'illusoria chiarezza della "gioia dell'anima" e lascia imprecisato il terreno su cui questo movimento si verifica. Il seguito dell'articolo del Dictionnaire, trattando della mistica cristiana del Medioevo, ci informa che "il significato attuale del termine, con l'accento sul carattere esperienziale e l'importanza conferita agli elementi e alle condizioni psicologiche che permettono di comprenderlo," compare per la prima volta all'inizio del ' $400 .{ }^{1}$ Nonostante poi l'articolo, ad opera di altri autori, si soffermi dettagliatamente sulle caratteristiche dell'esperienza mistica cristiana, sempre a proposito di mistica medievale si afferma che dell'esperienza in quanto tale "si ignora tutto," mentre l'unico indizio sono dei testi che riferiscono dei ricordi. E questi testi, prosegue l'articolo, sono sempre già il risultato di un confronto. Quando poi si passa ad altri testi meno personali, quali le prediche, le lettere, i trattati, il livello di redazione è quello del confronto delle idee ricevute e del linguaggio religioso di un luogo determinato. Sono affermazioni di estrema importanza, dalle quali però gli autori dell'articolo non traggono le conseguenze che si potrebbero trarre, e cioè che la mistica, se si guarda bene, non ha altro luogo che il linguaggio, e più precisamente il linguaggio in quanto già dato e cioè la scrittura. ${ }^{2}$

Mistica e scrittura costituiscono, e non solo nel Medioevo, un binomio inscindibile. Jean Leclercq, monaco benedettino egli stesso, dedica tutto un libro, L'anour des lettres et le désir de Dieu, a studiare il rapporto tra questi due sentimenti, per concludere che i monaci benedettini e cistercensi-San Bernardo in primis-che ci hanno lasciato le loro opere mistiche "non sono veramente divisi tra ricerca dell'arte e la ricerca di Dio, tra la retorica e l'esigenza di superamento insita nella loro vocazione, tra la grammatica e il desiderio escatologico. La retorica è diventata una parte di loro stessi” (169-70). Le af- 
fermazioni di Leclercq si riferiscono ovviamente ai monaci dotti della tradizione benedettina, a quelli che hanno studiato e scritto (anche se tutti i monaci che vogliono seguire la regola devono saper leggere). Tanto più significativo diventa allorá il confronto con l'altro filone della mistica medievale, quello rappresentato dalle donne, spesso non monache, di norma meno colte degli uomini e a volte addirittura analfabete. Angela da Foligno (1248-1309), terziaria francescana dopo essere stata moglie e madre, analfabeta, si sente spinta a dettare la sua esperienza a un frate francescano. Una dettatura difficile, sia dal punto di vista delle circostanze in cui avviene (il frate viene ripetutamente richiamato dai suoi superiori per la sconvenienza di questa privata registrazione della parole di una donna), sia da un punto di vista direi stilistico (ma già molto più che stilistico): Angela infatti non solo non si contenta di vivere la sua esperienza "mistica" in segreto, ineffabilmente, non è paga di una "gioia dell'anima" trasfigurante. A rischio suo e di altri, Angela detta: "Qua vero causa et quomodo ego indignus scriptor coactus, ut credo, a deo fuerim ad scribendum et predicta Christi fidelis omnino coacta fuerit ad dicendum, reperietur scriptum infra" (2). Ma c'è di più, come dicevo, perchè la scrittura del frate viene criticata come arida e oscura: "Et alia vice quando ego relegebam ei ut ipsa videret si ego bene scripseram, et ipsa respondit quod ego sicce et sine omni sapore loquebar; et ammirabatur de hoc. Et alia vice exposuit illa dicens: Per ista verba recordor que dixi tibi; sed est obscurissima scriptura; quia ista verba que legis michi non explicant illa que portant: ideo est scriptura obscura" (42). Altre volte Angela sembra ostentare una comprensione superiore del funzionamento della scrittura per eccellenza, la Sacra Scrittura: "intelligo ideo illud, quomodo facta est Scriptura divina; illud, quomodo facta est difficilis et facilis; illud, quomodo videtur dicere et contradicere" (238). "Le saint qui devient mystique reçoit une fonction scripturaire," ha scritto Michel De Cerleau (139). Alla funzione si accompagna, perfino in una donna analfabeta come Angela, la consapevolezza della stessa, consapevolezza che entra a costituire la stessa "esperienza mistica." In un passo del Liber de experientie, oscuro ma suggestivo, dopo aver detto che lo Spirito Santo le ha dichiarato che, se lei lo amerà ancora di più, le saranno date grazie più grandi di quelle date a San Francesco, la mistica dichiara: "In istis verbis cepi dubitare multum, et dixit an- 
ima sibi: Si tu esses spiritus sanctus, non diceres istud michi, quia non possum inde habere vanam gloriam. Et respondit: Modo cogita, si tu de omnibus istis potes habere unam vanam gloriam qua extollaris. Et exeas de istis verbis si potes. Et ego incepi et conata fui velle habere vanam gloriam, ut probarem si erat verum illud quod dixerat, et si erat spiritus sanctus. Et incepi respicere per vineas, ut exirem de illo, scilicet, de illa locutione" (48; il corsivo è mio).

Se l'esperienza mistica riveste intrinsecamente un carattere scritturale, si puó temporaneamente concludere almeno che mistica e poesia hanno in comune di essere sistemi di organizzazione del reale mediante la scrittura. Le ascendenze mistiche di Dante sono state abbondantemente indicate dagli studiosi, e del resto sono riconosciute nella lettera a Cangrande. A me però non interessa tanto sottolineare una similarità di nodi retorici, quanto piuttosto indicare una comune scaturigine, un modo di porsi davanti alla scrittura e quindi al mondo. Se il mistico è un credente che si costituisce come scrittore, il credente Dante è uno scrittore che dice "io" come il mistico. ${ }^{3}$ Per indicare l'atteggiarsi del mistico, la tradizione mistica ricorre a un termine chiave preso da San Paolo (Ef. 3.12): parrhesia, che in greco classico significa la libertà di prendere la parola nell'assemblea del popolo, privilegio del libero cittadino. Generalmente i commentatori non prendono in considerazione la connotazione langagière del termine, soffermandosi invece sul suo contenuto teologico: è l'Incarnazione che rende possible la parrhesia. A me sembra che in questo caso richiamarsi al valore originario del termine non equivale a erigere l'etimologia a metafisica, ma serve a illuminare un carattere della mistica anzi il carattere fondamentale della mistica, che finora è stato poco visto e poco studiato. ${ }^{4}$ Angela da Foligno, dopo aver affermato che la Scrittura "aliquid balbutit," proclama che "quamvis ego blasphemem dicendo et male dicendo illud quia non possum illud loqui," tuttavia "in illo manifestare Dei intelligo et habeo totam veritatem" (240), dunque in un certo senso asserisce, dettando, il proprio diritto a "rifare" la Sacra Scrittura, o per lo meno ad affiancarle un testo, il proprio, costruito sulla verità tanto quanto la parola di Dio. Così il prendere la parola di Dante o, piuttosto, il suo riprendere la parola dopo gli esperimenti giovanili, ${ }^{5}$ sgorga da una libertà di parola, parrhesia, ef- 
fetto della partecipazione alla grazia dell 'incarnazione, per cui non sarà semplicemente blasfemo dare al proprio poema la qualifica di "sacrato."

Sulla relazione tra i termini "poema" e "sacro," Angelo Jacomuzzi scrive delle pagine molto penetranti, là dove insiste sulla loro inscindibilità e sulla subordinazione, in un certo senso incontestabile, dell'aggettivo "sacro" al sostantivo "poema." Per Jacomuzzi, che si prefigge di ridurre lo iato tra ispirazione religiosa e poesia in Dante, opponendosi sia all'impostazione che diremo crociana, sia a quella di cui Singleton è il proponente più illustre, Dante "si pone al culmine della tradizione letteraria del medioevo latino e romanzo, raccogliendo in sè, nella figura dello scriba, la ricchezza vitale e l'attesa escatologica del tempo, e insieme, nella figura del poeta, l'idea di un operare artistico che è intrinsecamente artificio e di uno scrivere che è sempre un 'dictare,' che cerca la solidarietà del lettore nell'universo del discorso e non in quello del verisimile e della storia" (99). Jacomuzzi illustra l'interdipendenza dell'elemento "sacrale" e di quello poetico soprattutto attraverso il topos dell'ineffabilità. "L'indicibilità del fatto mistico della visione fonda . . . l'arbitrarietà del fatto linguistico, rivela la forma generale della Commedia come 'poema sacro' non nel momento della definizione contenutistica, ma in quello della definizione strutturale nella quale i due termini non si dispongono come solidali e convergenti, ma come opposti in tensione tra loro" (150). Pur essendo pienamente sottoscrivibile, questa formulazione va riaggiustata mediante due considerazioni che $\mathrm{mi}$ sembrano fondamentali: una riguarda il carattere dell'esperienza mistica, l'altra l'assetto retorico-formale del Paradiso, in particolare dell'ultimo canto.

Ho già accennato al carattere intrinsecamente scritturario dell'esperienza mistica. Resta da esplicitare una considerazione rimasta implicita nelle osservazioni precedenti e che meriterebbe di essere trattata più profondamente in altra sede. Dire che l'esperienza mistica è intrinsecamente scritturaria significa demitizzarne radicalmente il senso. Se da una parte il mistico è la percezione intuitiva di un aldilà dell'esperienza empirica normale (per attenerci alla definizione tradizionale del Dictionnaire), e dall'altra è una ricerca di parole, un'ostinata lotta con l'angelo in vista di una ri-scrittura del già scritto, allora questo aldilà si riduce a un punto, uno zero, un’ori- 
gine fuori dal linguaggio ma da sempre intrappolata in esso. Un aldilà demitizzato, cioè radicalmente sciolto dal mito che lo nomina e come lo avvolge. L'esperienza mistica è questo divisorio, questa superficie a due facce, questa lamina che vibra a contatto dello zero, dell'innominabile, ${ }^{6}$ del sono-quel-che sono; questa superficiale scrittura tremante, che si attraversa per risolversi nel desolato giubilo del silenzio. Il linguaggio che così si produce, appoggiato per così dire all'indicibile, è un linguaggio "falso" (il quasi pro truffis di Angela da Foligno), staccato da qualsiasi pretesa di corrispondenza alle cose. Afferma De Certeau: "au lieu de supposer qu'il y a quelque part du mensonge et qu'à le dépister et déloger on peut restaurer une vérité (et une innocence?) du langage, le préalable mystique pose un acte qui conduit à utiliser le langage tout entier comme menteur" (241). Non so se è il caso di sottolineare ancora quanto il "falso" e il "finto" si tocchino, quanto la "falsità" intrinseca all'espressione mistica non sia di lega diversa dalla fittività dell'espressione poetica. C'è però una differenza: la falsità del linguaggio mistico come la sua dolorosa novità si configurano come tali rispetto a una autorità che le precede. Il linguaggio mistico è un controcanto a una scrittura autorevole, nel nostro caso la Sacra Scrittura. Il mistico pretende di fare lo stesso discorso della Scrittura (e dell'autorità ecclesiastica che lo interpreta), ma si arroga il diritto e l'autorità di farlo in maniera diversa. Rimando alla conclusione la precisazione inevitabile riguardo alla direzione del linguaggio dantesco.

Vorrei adesso soffermarmi brevemente su alcuni aspetti retoricoformali del Paradiso, per vedere se si trovano qui delle indicazioni sul luogo a cui assegnare la scrittura di Dante. Il topos dell'ineffabilità, così frequente nella terza cantica, non è univocamente interpretabile in senso mistico. Jacomuzzi insiste sul fatto che le dichiarazioni di ineffabilità non hanno come funzione "l'esaltazione del personaggio o dell'accadimento"; "ciò che si evidenzia ... non è propriamente la trascendenza paradisiaca della visione, ma la eccezionalità della situazione psicologica e linguistica, il ponderoso tema, il limite ultimo toccato dall'artista, la condizione critica, insomma, dell'invenzione poetica" (124-5). Se si leggono due tra i più importanti di questi passi, non si può non essere d'accordo sull'importanza che in essi riveste quella che si potrebbe chiamare la metaretorica di Dante.

"Apri li occhi e riguarda qual son io: 
tu hai vedute cose, che possente se fatto a sostener lo riso mio."

lo era come quei che si risente di visione oblita e che s'ingegna indarno di ridurlasi a la mente,

quand 'io udi' questa proferta, degna di tanto grato, che mai non si stingue del libro che 'l preterito rassegna.

Se mo sonasser tutte quelle lingue che Polimnia con le suore fero del latte lor dolcissimo più pingue,

per aiutarmi, al millesmo del vero non si verría, cantando il santo riso e quanto il santo aspetto il facea mero;

e cosí, figurando il paradiso, convien saltar lo sacrato poema, come chi trova suo cammin riciso.

Ma chi pensasse il ponderoso tema e l'omero mortal che se ne carca, nol biasmerebbe se sott'esso trema:

non è pileggio da picciola barca quel che fendendo va l'ardita prora, né da nocchier ch'a se medesmo parca.

(Par. 23.46-69)

Se quanto infino a qui di lei si dice, fosse conchiuso tutto in una loda poca sarebbe a fornir questa vice.

La bellezza ch'io vidi si trasmoda non pur di là da noi, ma certo io credo che solo il suo fattor tutta la goda.

Da questo passo vinto mi concedo piú che già mai da punto di suo tema soprato fosse comico o tragedo;

ché, come sole in viso che piú trema, cosí lo rimembrar del dolce riso la mente mia da me medesmo scema.

Dal primo giorno ch'i' vidi il suo viso in questa vita, infino a questa vista, non m'è il seguire al mio cantar preciso;

ma or convien che mio seguir desista piú dietro a sua bellezza, poetando. come a l'ultimo suo ciascuno artista. 
È vero, come afferma Jacomuzzi, che "solo una lettura arresa a priori alle abitudini esegetiche o ai luoghi comuni della parafrasi critica può avvertire come prevalente in questi passi l'eco verbale di un'esperienza mistica" (121). È vero anche però, dal mio punto di vista, che "l'eco verbale di un'esperienza mistica" non è necessariamente (anzi, lo è raramente, e comunque questo fenomeno non ci interessa) eco verbale di un'emozione psico-fisiologica, e ancor meno di una "visione" letteralmente intesa: se di eco verbale si tratta (ma ormai queste parole sono inadeguate), allora è l'eco di uno sforzo, già all'origine del linguaggio, di costeggiare da vicino l'aldilà del linguaggio stesso. Jacomuzzi continua: "Il loro oggetto proprio non è tanto un accadimento, la visione oblita e indicibile, quanto il poema, nella sua definizione-il sacrato poema-e nelle sue metafore-l'ardita prora, il cantar; o, più precisamente, il modo fictivus, transumptivus, poeticus insomma, della trattazione, così energicamente richiamato nei rinvii letterari e non mistici e sacrali" (121-2). Non si può non riconoscere che in questi passi sia sottolineata la "fatica dell'invenzione" (122). La drammaticità dei rinvii letterari è però meno evidente quando si rifletta sull'utilizzo indiscriminato, proprio al medioevo, degli autori di qualsiasi fede. Leclercq, nell'opera citata, non si stanca di sottolineare quanto questa pratica fosse diffusa negli ambienti monastici-gli stessi da cui nasce la letteratura mistica: nemmeno l'Ars amandi sfugge all'integralismo, per dir così, dei santi medievali. Quando si oppongono in maniera irriducibile "letteratura" e "religione" a proposito di fenomeni medievali, si rischia di attribuire al passato categorie di pensiero che sono invece solo le nostre.

Prima di concludere sul modo in cui la consapevolezza diciamo artistica di Dante agisce sulle ragioni della sua scrittura, vorrei però esaminare brevemente altri due aspetti formali che mi pare contribuiscano ad accentuare ulteriormente il carattere mistico, nel senso che sono venuta precisando, del Paradiso. Si tratta rispettivamente del topos del vedere e di quella che è stata diversamente chiamata trasparenza, allusività, estenuazione, incorporeità della terza cantica. Secondo Jacomuzzi, 'il 'vidi' che segna con variata intensità e frequenza il racconto della visione lungo tutto l'arco delle tre cantiche ... sta a indicare il momento più alto della mitizzazione, la coincidenza di visione e invenzione, rivelazione e linguaggio, e sottolinea 
energicamente la potenza e la sufficienza della rappresentazione" (150-1). Mi riservo di analizzare questo topos in altra sede. Ma mi pare di poter affermare che, almeno nel Paradiso, l'insistenza sul vedere si ribalta di fatto in un'assenza di visione. Nell'Inferno avveniva che l'oggetto della visione incalzasse la rappresentazione dell'atto del vedere:

Come le rane innanzi alla nimica biscia per l'acqua si dileguan tutte, fin ch'a la terra ciascuna s'abbica,

vid'io piú di mille anime distrutte fuggir cosí dinanzi ad un ch'al passo passava Stige con le piante asciutte.

(Inf. 9.76-81)

Nella terza Cantica il vedere si esplicita ben altrimenti:

Beatrice tutta ne l'eterne rote fissa con li occhi stava; ed io in lei le luci fissi, di là sú remote.

Nel suo aspetto tal dentro mi fei, qual si fe' Glauco nel gustar de l'erba che 'l fe' consorto in mar de li altri dei.

Trasumanar significar per verba non si poría; però l'essemplo basti a cui esperienza grazia serba.

(Par. 1.64-72)

Quel che Dante "vede" in Beatrice è questo: una similitudine che squalifica l'unicità dichiarata dell'esperienza, incalzata da una riflessione metaretorica che ci fa toccare i limiti del linguaggio. II Sapegno qui commenta: "Il vertice della tensione poetica si colloca, per così dire, al di là della rappresentazione propriamente detta, nell'entusiasmo che accompagna la rievocazione di un'esperienza ineffabile e nello sforzo che il poeta compie per renderne partecipe il lettore" (nota a Par. 1.66). Ma si tratta davvero della "rievocazione di un'esperienza ineffabile," oppure l'esperienza è precisamente quella dell'impossibilità della visione, della sconfitta del linguaggio?' Il canto 30 del Paradiso, dopo la preghiera di San Bernardo, non è che un susseguirsi di annunci di visione, annunci che vengono o immediatamente ribaltati nell'impossibilità di rappresentare, o seguiti da un oggetto della visione che per definirsi deve ricorrere ai modi di un linguaggio preesistente: 
Nel suo profondo vidi che s'interna legato con amore in un volume, ciò che per l'universo di squaderna; sustanze e accidenti e lor costume, quasi conflati insieme, per tal modo che ciò ch'i' dico è un semplice lume.

(Par. 33.85-90)

Non si può dire che questa rappresentazione sia molto originale, dato che si limita a riprendere, fin nella terminologia, concetti artistotelici e scolastici. E più avanti:

$\mathrm{Ne}$ la profonda e chiara sussistenza de l'alto lume parvermi tre giri di tre colori e d'una contenenza;

e l'un da l'altro come iri da iri parea reflesso, e 'l terzo parea foco che quinci e quindi igualmente si spiri.

Oh quanto è corto il dire e come fioco al mio concetto! e questo, a quel ch'i' vidi, è tanto, che non basta a dicer "poco."

Anche qui, la dichiarazione di impossibilità ad esprimere incalza una rappresentazione che non fa che riformulare pallidamente, sotto una leggera patina di immagini, un concetto tratto da un linguaggio presistente (il simbolo niceno: lumen de lumine). Vediamo ancora i versi $127-132$ :

Quella circulazion che sí concetta pareva in te come lume reflesso, da li occhi miei alquanto circunspetta, dentro da sé, del suo colore stesso, mi parve pinta de la nostra effige; per che 'l mio viso in lei tutto era messo.

Sconfitta della rappresentazione nel momento stesso in cui il poeta intende metterla in gioco: L'Incarnazione è detta in un linguaggio che appena si discosta dal linguaggio teologico. Anche per questo verso-la visione che "vede," ma solo quello che è già stato visto-viene confermata la scaturigine "mistica" del Paradiso. Dire le stesse cose, ma dirle in maniera "falsa," modo fictivo, quasi pro truffis!

E veniamo brevemente all'“incorporeità" della poesia del Paradi- 
so. La critica oscilla tra la svalutazione e la celebrazione, poli solo in apparenza opposti di un unico presupposto idelogico che intende la Commedia come rappresentazione mimetica della realtà. Cosi, mentre da una parte il Paradiso viene ritenuto poeticamente inferiore rispetto alla trionfale rappresentatività dell'Inferno, dall'altra vienc giustificato in quanto attinente ad una indefinibile pocsia degli affetti soprannaturali (impostazione, quest'ultima, che fa irresistibilmente pensare älla dottrina medievale e dantesca dei corpi aerei). A me sembra che la definizione globale più appropriata (ammesso che sia legittimo fare tali generalizzazioni) della poesia del Paradiso sia quella di poesia "lasca," poesia dalla maglie larghe, allentata rispetto alla mimesi, appoggiata al silenzio. Prendiamo un passaggio che tra l'altro si riferisce anche al topos del vedere:

Come a raggio di sol che puro mei per fratta nube già prato di fiori vider, coverti d'ombra, li occhi miei;

vid io cosí piú turbe di splendori, folgorate di sú da raggi ardenti, senza veder principio di fulgóri.

(Par. 23.79-84)

Ecco Dante, il sommo artefice della lingua, passare in due terzine dalla precisa articolazione verbale di un brano di esperienza sensibile ad un balbettio ostinato in cui il silenzio è prepotentemente in agguato. Ecco una terzina, la seconda, che mentre si dichiara rappresentazione di un oggetto della vista, si frantuma per così dire in un cumulo di vocaboli, tutti connotanti "luce" (splendori, folgorate, raggi, ardenti, fulgori), che stentano a formare una immagine. Una specie di vittoria di Pirro: vince la parola, o piuttosto la scrittura, per ora, ma a che prezzo! Questo toccare i limiti del linguaggio, questo cedere le armi, questo "désespoir très proche du ravissement" (Blanchot 10), non sono altro che l'esperienza mistica, vibrazione nel linguaggio del silenzio dell'origine.

Dante "mistico," allora? Anche accettando una ridefinizione di esperienza mistica in cui sia integrato come essenziale l'aspetto scritturario, è ovvio che Dante non tollera di essere semplicemente assimilato agli scrittori spirituali del medioevo. Non perchè, come tradizionalmente la critica sottolinea, egli sia troppo preso dal "terrestre," ma per la coscienza linguistico-retorica che lo sorregge. Non 
a caso Dante nasce alla scrittura prima di approdare alla mistica, e fin nella Commedia si può rintracciare un percorso che è di superamento e di distacco nei confronti di modi poetici anteriori, meno vicini alle regioni del silenzio. Abbiamo visto che anche il "mistico puro" è consapevole, intrinsecamente, costitutivamente, della artificiosità della sua scrittura, proprio mentre si dice implacabilmente spinto a parlare. Ma forse è più del mistico-poeta disposto a "terminare," pur nel terrore, perchè si affida "subito" a un silenzio culturalmente connotato in maniera positiva. 11 mistico-poeta (ma forse in questa categoria non c'è che Dante a rientrare) oscilla tra il silenzio e un linguaggio che, per il fatto di essere così coscientemente il prodotto di una ricerca, di un'arte, rischia di scollarsi dalle ragioni ultime, vivendo per così dire da solo, di una vita tragicamente precaria. Come si ripete volentieri, Dante sta a cavallo tra il medioevo e l'età moderna, Ulisse che per poco non soccombe alla follia del proprio volo: da una parte, tenuto ancora saldamente da un'adesione profonda (la "fede") ad una particolare definizione culturale di "aldilà" nel senso di ragione ultima, origine; dall'altra, follemente, coscientemente aggrappato alla propria "orazion picciola" nella quale rischia di consumarsi, lingua fatua e frodolente. ${ }^{8}$ Questo bilico è precisamente la condizione che rende possibile la Commedia.

Vorrei citare per concludere questo brano di Mallarmé (66):

Oui, je le sais, nous ne sommes que des vaines formes de la matière, mais bien sublimes pour avoir inventé Dieu et notre âme. Si sublimes, mon ami!, que je veux me donner ce spectacle de la matière, ayant conscience d'être, et cependant, s'élançant forcenément dans le rêve qu'elle sait n'être pas, chantant l'Ame et toutes les divines impressions pareilles qui se sont amassées en nous depuis les premiers âges, et proclamant, devant le Rien qu'est la vérité, ces glorieux mensonges!

Il libro di Mallarmé è, come sappiamo, impossible. Quello di Dante, nella sua "disagguaglianza" (Par. 15.82), è riuscito per tre cantiche a superare ogni "passo forte" e sta quasi blasfemo, al di là del vero e del falso, a trascinarci nella propria fragilità.

St. John's University 


\section{NOTE}

- 11 presente saggio è una versione riveduta di una conferenza fatta a Yale nell'ottobre del 1985.

I Si tratta di una precisazione importante: quando si parla di mistica oggi, si tende a caricare il termine del peso psicologico di cui lo hanno accresciuto gli scrittori spagnoli del ' 500 .

2 Si potrà obbiettare che la gioia dell'anima esiste: può darsi, ma questo fenomeno riguarda la psicologia e la neurobiologia, non la poesia e neanche la mistica.

3 Questa affermazione è ovviamente da modificare, e lo sarà nel seguito dell'articolo.

4 L'eccezione, e di grande rilievo, è rappresentata dagli studi di Michel De Certeau, prematuramente scomparso agli inizi del 1986.

5 Il luogo obbligato qui è il passo della "mirabile visione, ne la quale io vidi cose che mi fecero proporre di non dire più di questa benedetta infino a tanto che io potesse più degnamente trattare di lci" (V.N. 42.1).

6 Cf. Samuel Beckett, The Unnamable 134: "l'm in the middle, I'm the partition. I've two surfaces and no thickness, perhaps that's what I feel, myself vibrating."

7 Non si deve trarre da questo, come qualcuno ha fatto, la conclusione che l'arrendersi di Dante di fronte all'oggetto della visione è un'altra spia dell'assenza di "autentica" esperienza mistica in Dante: anche i mistici canonizzati, se a volte descrivono qualche "visione," sottolineano che queste non hanno niente a che fare con l'essenza della loro conoscenza di Dio.

$8 \mathrm{Mi}$ ispiro qui alla bella analisi che del canto 26 dell'Inferno fa Giuseppe Mazzotta nei suo libro Dante, Poet of the Desert.

\section{OPERE CITATE}

Alighieri, Dante. La Divina Commedia. A cura di N. Sapegno. 3 vols. Firenze: La Nuova Italia, 1968.

Le Opere di Dante. Firenze: Società Dantesca Italiana, 1960.

Beckett, Samuel. The Unnamable. New York: Grove Press, 1958.

Blanchot, Maurice. Le livre à venir. Paris: Gallimard, 1959.

Croce, Benedetto. La poesia di Dante. Bari: Laterza, 1921.

Da Foligno, Angela. Le livre de l'expérience des vrais fideles. Texte latin publié d'après le manuscrit d'Assise par M.-J. Ferré. Paris: E. Droz, 1927.

De Certeau, Michel. La fable mystique. Paris: Gallimard, 1982.

Dictionnaire de spiritualité ascétique et mystique. Vol. 10. Paris: G. Bcauchesne, 1977.

Fallani, Giovanni. Dante poeta teologo. Milano: Marzorati, 1965.

Getto, Giovanni. Aspetti della poesia di Dante. Firenze: Sansoni, 1965.

Jacomuzzi, Angelo. L'imago al cerchio: invenzione e visione nella Divina Commedia. Milano: Silava, 1968. 
Leclercq, Jean. L'amour des lettres et le désir de Dieu. Paris: Éditions du Cerf, 1957.

Mallarmé, Stéphane. Propos sur la poésie. Monaco: Éditions du Rocher, 1953. Mazzotta, Giuseppe. Dante, Poet of the Desert. Princeton: Princeton UP, 1979.

Nardi, Bruno. "Dante profeta." Dante e la cultura medievale. Bari: Laterza, 1949.

Sarolli, Gian Roberto. Prolegomena alla Divina Commedia. Firenze: Olschki, 1971.

Singleton, Charles S. Commedia: Elements of Structure. Cambridge: Harvard UP, 1954. 